In Cres. Vol. $4 N^{\circ} 1: p p .133-146,2013$

\title{
ESTILO DE VIDA Y FACTORES BIOSOCIOCULTURALES DEL ADULTO MAYOR DEL ASENTAMIENTO HUMANO SAN JUAN-CHIMBOTE, 2012*
}

\author{
LIFE STYLE AND SOCIOCULTURAL FACTORS OF THE ELDERLY PEOPLE \\ IN THE HUMAN SETTLEMEN SAN JUAN-CHIMBOTE, 2012.
}

\author{
María Isabel Sare Vega', \\ Susana Cabanillas De la Cruz $^{2}$
}

\begin{abstract}
RESUMEN
El presente trabajo de investigación de tipo cuantitativo, descriptivo correlacional, de corte transversal, tuvo como objetivo determinar la relación entre el estilo de vida y los factores biosocioculturales del adulto mayor del asentamiento humano "San Juan"-Chimbote, 2012. La muestra estuvo conformada por 84 personas adultas mayores de ambos sexos. La recolección de datos se efectuó a través de las visitas domiciliarias, teniendo como instrumento la escala del estilo de vida y el cuestionario semi-estructurado sobre factores biosocioculturales. El análisis de datos se efectuó mediante la prueba de independencia del Chi cuadrado $\left(\mathrm{X}^{2}\right)$ con el $95 \%$ de confiabilidad y significancia de $\mathrm{p}<0,05$. La mayoría de los adultos mayores tiene un estilo de vida no saludable; sin embargo, existe un porcentaje significativo con estilo de vida saludable. Respecto a los factores biosocioculturales, un poco más de la mitad fueron masculinos; la mayoría tiene grado de instrucción primaria completa e incompleta; cuentan con un ingreso económico de 100 a 599 Nuevos Soles; son viudos y con unión libre; más de la mitad son evangélicos. No existe relación significativa entre el estilo de vida y los factores biosocioculturales: sexo, grado de instrucción, religión, estado civil e ingreso económico; pero sí existe relación significativa con el factor social de ocupación de los adultos mayores.
\end{abstract}

PALABRAS CLAVE: Estilo de vida, factores biosocioculturales y adulto mayor.

* Recibido: 30 de marzo del 2013; aprobado: 16 de abril del 2013.

1 Licenciada en Enfermería.

2 Magister en Enfermería. Docente de Enfermería. ULADECH-Católica. 


\begin{abstract}
This non-experimental research of descriptive corelational design aimed to establish the relationship between life style and sociocultural factors of the elderly people in the human settlemen San Juan-Chimbote. The sample consisted of 84 elderly people, males and females, who were visited at home. The instruments used were the life style scale and sociocultural factors questionnaire. The data was statistically analyzed by means of the Chi Square test for independence with a $5 \%$ significance level and $95 \%$ of reliability. The study came to the following conclusions: Most of elderly people present unhealthy lifestyle, however, a significant number of them present healthy one. Respect to the biosociocultural factors, most are male. Most have completed and non-completed primary education. Their salary range from 100 and 599 Nuevos Soles monthly. Most of them are widowed and widowers. More than half are evangelists. There is no significant relationship between lifestyle and the sociocultural factors: sex, education level, religion, marital status, and income; but there is significant relation between elderly people`s occupation.
\end{abstract}

KEY WORDS: Lifestyle, sociocultural factors, elderly people.

\title{
I. INTRODUCCIÓN
}

La presente investigación se realizó con la finalidad de determinar la relación entre el estilo de vida y los factores biosocioculturales del adulto mayor del asentamiento humano (A.H.) San Juan, del distrito de Chimbote, provincia de Santa, departamento de Ancash.

Esta investigación se fundamenta en las bases conceptuales del estilo de vida y factores biosocioculturales de la persona adulta. El estilo de vida es una forma de vida que se basa en patrones de comportamiento identificables, determinados por la interacción entre las características personales individuales, las interacciones sociales y las condiciones de vida socioeconómicas y ambientales. Desde una perspectiva integral, es necesario considerar los estilos de vida como parte de una dimensión colectiva y social, que comprende tres aspectos interrelacionados: el material, el social y el ideológico. ${ }^{1}$

Los factores biosocioculturales son expresados colectivamente mediante la construcción de estilos de vida distintos, localizados, fundamentalmente, en tiempo, o en espacios intersticiales de la vida, conformados por la edad, el sexo, hábitos, costumbres, modas, valores existentes y dominantes en cada momento y en cada comunidad. Estos factores enumerados tienen como característica común que son aprendidos y, por tanto, algunos pueden ser modificables a lo largo de toda la vida. ${ }^{2}$ 
La preservación de la salud física y mental constituye una responsabilidad individual, con independencia de las acciones dirigidas a su conservación en el ciudadano y representa un deber cívico para evitar añadir una carga económica o sentimental, a corto o lejano plazo, a la familia o a la propia sociedad, dando la posibilidad de alcanzar una vejez sana, portadora de ricas y ejemplarizantes experiencias para los más jóvenes. Tal legado es un estilo de vida. ${ }^{1}$

La enfermedad cardiovascular, los derrames cerebrales, las enfermedades crónico-degenerativas, el consumo de sustancias nocivas, son las primeras causas de muerte en la mayoría de los países de América. Las tasas de morbimortalidad siguen en aumento en la población y están subiendo por todo el hemisferio. Se estima que en América, en menos de 20 años, una de cada 10 personas en el hemisferio Oeste tendrá diabetes. ${ }^{3}$

La salud de la población peruana es un reflejo de su realidad social: se ha alcanzado una importante mejora en algunos indicadores de salud del país; sin embargo, las grandes diferencias que enmascaran los promedios nacionales ocultan las inequidades existentes en la salud. La mayor o menor probabilidad de muerte y enfermedad están en función de factores como el estrato socioeconómico, la condición de ruralismo, el género y el nivel educativo en los que se encuentren las personas y las comunidades. ${ }^{4}$

A esta realidad no escapa el adulto mayor que reside en el sector del A.H. San Juan, que pertenece al distrito de Chimbote, provincia del Santa en la costa norte del Perú. Esta comunidad pertenece a la jurisdicción de la Red Pacifico Norte - Microred Miraflores Alto, el puesto de salud San Juan. El AA.HH. San Juan limita por el Norte con el parque recreacional San Juan, por el sur con la Avenida Los Pescadores, por el este A.H. Villa España y por el Oeste con Av. Pardo. ${ }^{5}$

El A.H. San Juan es una comunidad urbanomarginal, cuenta con servicios básicos y tiene una población de adultos mayores de 84 . La condición socioeconómica es de pobreza y pobreza extrema, existe un alto índice de desempleo, subempleo, así como problemas sociales de delincuencia y drogadicción. Las principales actividades a que se dedica la población son: obreros de fábricas pesqueras, agricultura; también hay adultos mayores que se dedican al comercio ambulatorio de frutas, verduras, etc. ${ }^{5}$

El adulto mayor recibe atención de salud en el Puesto de Salud San Juan, donde no se cuenta con un programa activo para el adulto mayor; pero de manera periódica se realizan campañas de promoción y prevención de la salud del adulto 
en su jurisdicción, las cuales incluyen consulta médica, cuidados de enfermería preventiva promocional, educación, consejería, consulta obstétrica. ${ }^{5}$

Frente a lo expuesto, se consideró pertinente la realización de la presente investigación.

\section{PROBLEMA}

¿Existe relación entre el estilo de vida y los factores biosocioculturales del adulto mayor del A.H. San Juan, en Chimbote, 2012?

\section{OBJETIVOS}

El objetivo general fue determinar la relación entre el estilo de vida y los factores biosocioculturales del adulto mayor del A.H. San Juan, Chimbote.

Los objetivos específicos:

a) Valorar el estilo de vida del adulto mayor del A.H. San Juan, Chimbote;

b) Identificar los factores biosocioculturales: sexo, grado de instrucción, religión, estado civil, ocupación e ingreso económico del adulto mayor.

La investigación se fundamenta en las bases conceptuales, estilo de vida y factores biosocioculturales de la persona.

Según Lalonde en Tejera ${ }^{7}$, los estilos de vida son la forma de vivir de las personas, que, al igual que el medio ambiente, están determinados por un conjunto de factores (físicos, sociales o laborales) que dependen tanto del individuo como del complejo entorno que le rodea, dando lugar a estilos de vida mas o menos saludables.

El estilo de vida es un modo de vida individual, es la forma de vivir de las personas. Se relaciona estrechamente con la esfera conductual y motivacional del ser humano y, por tanto, alude a la forma personal en que el hombre se realiza como ser social, en condiciones concretas y particulares. En la actualidad, se considera que la familia como grupo particular, con condiciones de vida similares, posee un estilo de vida propio que determina la salud del grupo familiar y sus miembros, por lo que se concibe, además, la categoría de estilo de vida familiar. ${ }^{7}$

En cuanto a los factores biosocioculturales de la persona, para el presente estudio, se han considerado: la edad, sexo, estado civil, grado de instrucción, ocupación, ingreso económico y religión. Ciertos factores tienen influencia indirecta sobre las conductas de salud, a través de los factores cognitivo- 
perceptuales. Estos factores pueden ser demográficos: se proponen que la edad, sexo, raza étnica, educación procedencia e ingreso económico tienen influencia indirecta a través de su impacto en el establecimiento de conductas de salud. ${ }^{8}$

\section{METODOLOGÍA}

Este estudio es de tipo cuantitativo, de corte tranversal, descriptico correlacional. ${ }^{9,10}$

La población muestral estuvo constituida por 84 adulto que residen en el A.H. San Juan en Chimbote y que cumplieron con los criterios de inclusión.

La variable Estilo de vida del adulto mayor está definida como un conjunto de patrones conductuales que una persona en concreto pone en práctica de manera consistente y mantenida en su vida cotidiana y que puede ser pertinente para el mantenimiento de su salud o que lo coloca en situación de riesgo para la enfermedad. Se utilizó la escala nominal, estilo de visa saludable y estilo de vida no saludable. ${ }^{11}$

La variable Factores biosocioculturales es definida como características o circunstancias detectables en la persona, que pueden ser no modificables. Los factores considerados son: edad, estado civil, escolaridad, ingreso económico, ocupación, sexo, religión. ${ }^{12}$

Los datos fueron procesados en el paquete SPSS/info/software, versión 18.0. Se presentaron los datos en tablas simples y de doble entrada. Para establecer la relación entre variables de estudio se utilizó la prueba de independencia de criterios Chi cuadrado con el $95 \%$ de confiabilidad y significancia de $\mathrm{p}<0,05$.

\section{RESULTADOS Y DISCUSIÓN}

Tabla 01

ESTILO DE VIDA DE LAS PERSONAS ADULTAS MAYORES

DEL A.H. SAN JUAN, CHIMBOTE, 2012

\begin{tabular}{lcc}
\hline Estilo de vida & Frecuencia & Porcentaje \\
\hline Saludable & 38 & 45,0 \\
No saludable & 46 & 55,0 \\
\hline Total & 84 & 100,0 \\
\hline
\end{tabular}

Fuente: Escala de estilo de vida elaborada por: Walker, Sechrist y Pender, modificado por: Díaz, R; Marquéz, E; Delgado, R. aplicado a las personas adultas mayores del A.H. San Juan, febrero 2012. 
Tabla 02

FACTORES BIOSOCIOCULTURALES DE LAS PERSONAS ADULTAS MAYORES

\begin{tabular}{|c|c|c|}
\hline \multicolumn{3}{|c|}{ FACTORES BIOLÓGICOS } \\
\hline Sexo & $\mathbf{N}$ & $\%$ \\
\hline Masculino & 44 & 52,4 \\
\hline Femenino & 40 & 47,6 \\
\hline Total & 84 & 100,0 \\
\hline \multicolumn{3}{|c|}{ FACTORES CULTURALES } \\
\hline Grado de instrucción & $\mathbf{N}$ & $\%$ \\
\hline Analfabeto(a) & 30 & 35,7 \\
\hline Primaria incompleta & 36 & 42,9 \\
\hline Primaria completa & 18 & 21,4 \\
\hline Secundaria incompleta & 0 & 0,0 \\
\hline Secundaria completa & 0 & 0,0 \\
\hline Superior incompleta & 0 & 0,0 \\
\hline Superior completa & 0 & 0,0 \\
\hline Total & 84 & 100,0 \\
\hline Religión & $\mathbf{N}$ & $\%$ \\
\hline Católico(a) & 35 & 41,7 \\
\hline Evangélico(a) & 49 & 58,3 \\
\hline Total & 84 & 100,0 \\
\hline \multicolumn{3}{|c|}{ FACTORES SOCIALES } \\
\hline Estado civil & $\mathbf{N}$ & $\%$ \\
\hline Soltero(a) & 4 & 4,8 \\
\hline Casado(a) & 12 & 14,3 \\
\hline Viudo(a) & 23 & 27,4 \\
\hline Unión libre & 38 & 45,2 \\
\hline Separado(a) & 7 & 8,3 \\
\hline Total & 84 & 100,0 \\
\hline Ocupación & $\mathbf{N}$ & $\%$ \\
\hline Obrero(a) & 1 & 1,2 \\
\hline Empleado(a) & 0 & 0,0 \\
\hline Ama de casa & 25 & 29,8 \\
\hline Estudiante & 0 & 0,0 \\
\hline Agricultor & 16 & 19,0 \\
\hline Otras & 42 & 50,0 \\
\hline Total & 84 & 100,0 \\
\hline
\end{tabular}


Tabla 02 (Cont.)

\begin{tabular}{lrr}
\hline Ingreso económico (soles) & N & $\%$ \\
Menos de 100 & 4 & 4,8 \\
De 100 a 599 & 80 & 95,2 \\
De 600 a 1000 & 0 & 0,0 \\
Más de 1000 & 0 & 0,0 \\
Total & 84 & 100,0 \\
\hline
\end{tabular}

Fuente: Cuestionario de factores biosocioculturales elaborada por: Walker, Sechrist y Pender, modificado por: Diaz, R; Marquéz E; Delgado R. aplicado a las personas adultas mayores del AA.AH. San Juan, febrero 2012.

\section{TABLA 01}

Del 100\%(84) de adultos mayores que participaron en el estudio, el 55\% (46) tienen un estilo de vida no saludable y un $45 \%$ (38), saludable.

\section{TABLA 02}

El 52,4\% (44) de los adultos mayores son de sexo masculino. Con respecto a los factores culturales, el $42,9 \%$ (36) tiene primaria incompleta; en cuanto a la religión, el mayor porcentaje $(58,3 \%, 49)$ son evangélicos. En lo referente a los factores sociales, el 45,2\% (38) es de estado civil unión libre, el 50\% (42) tiene otras ocupaciones y un 95,2\% (80) tiene un ingreso económico de 100 a 599 nuevos soles.

Tabla 03

FACTORES BIOLÓGICOS Y ESTILO DE VIDA DE LAS PERSONAS ADULTAS MAYORES

\begin{tabular}{|c|c|c|c|c|c|c|}
\hline \multirow{3}{*}{ Sexo } & \multicolumn{4}{|c|}{ Estilo de vida } & & \\
\hline & \multicolumn{2}{|c|}{ Saludable } & \multicolumn{2}{|c|}{ No saludable } & \multicolumn{2}{|c|}{ Total } \\
\hline & $\mathrm{n}$ & $\%$ & $\mathbf{n}$ & $\%$ & $\mathbf{n}$ & $\%$ \\
\hline Masculino & 16 & 19,0 & 28 & 33,3 & 44 & 52,4 \\
\hline Femenino & 22 & 26,2 & 18 & 21,4 & 40 & 47,6 \\
\hline Total & 38 & 45,2 & 46 & 54,8 & 84 & 100,0 \\
\hline \multicolumn{7}{|c|}{$\begin{array}{c}\chi^{2}=2,937 ; 1 \mathrm{gl} \\
\mathrm{P}=0,087>0,05 \\
\text { No existe relación signific }\end{array}$} \\
\hline
\end{tabular}

Fuente: Cuestionario de factores biosocioculturales y escala de estilo de vida elaborada por: Walker, Sechrist y Pender, modificado por: Diaz, R; Marquéz E; Delgado R. aplicado a las personas adultas mayores del AA.HA. San Juan, febrero 2012 . 


\section{TABLA 03}

Se muestra la relación entre los factores culturales y el estilo de vida. Al relacionar el grado de instrucción con el estilo de vida utilizando el estadístico Chi-cuadrado de independencia $\left(\chi^{2}=1,384 ; 2 \mathrm{gl} \mathrm{y} \mathrm{p}>0,05\right)$ encontramos que no existe relación estadísticamente significativa entre ambas variables. Así mismo, muestra la relación entre la religión y estilo de vida, haciendo uso del estadístico Chi-cuadrado de independencia $\left(\chi^{2}=2,905 ; 1 \mathrm{gl} \mathrm{y} \mathrm{p}>0,05\right)$. Se encontró que no existe relación estadísticamente significativa entre la religión y el estilo de vida.

Tabla 04

FACTORES CULTURALES Y ESTILO DE VIDA DE LAS PERSONAS ADULTAS MAYORES

\begin{tabular}{|c|c|c|c|c|c|c|}
\hline \multirow{3}{*}{$\begin{array}{l}\text { Grado de } \\
\text { instrucción }\end{array}$} & \multicolumn{4}{|c|}{ Estilo de vida } & & \\
\hline & \multicolumn{2}{|c|}{ Saludable } & \multicolumn{2}{|c|}{ No saludable } & \multicolumn{2}{|c|}{ Total } \\
\hline & $\mathrm{n}$ & $\%$ & $\mathbf{n}$ & $\%$ & $\mathbf{n}$ & $\%$ \\
\hline Analfabeto & 14 & 16,7 & 16 & 19,0 & 30 & 35,7 \\
\hline Primaria incompleta & 18 & 21,4 & 18 & 21,4 & 36 & 42,9 \\
\hline Primaria Completa & 6 & 7,1 & 12 & 14,3 & 18 & 21,4 \\
\hline Total & 38 & 45,2 & 46 & 54,8 & 84 & 100,0 \\
\hline
\end{tabular}

\begin{tabular}{|c|c|c|c|c|c|c|}
\hline \multirow{3}{*}{ Religión } & \multicolumn{4}{|c|}{ Estilo de vida } & & \\
\hline & \multicolumn{2}{|c|}{ Saludable } & \multicolumn{2}{|c|}{ No saludable } & \multicolumn{2}{|c|}{ Total } \\
\hline & $\mathrm{n}$ & $\%$ & $\mathbf{n}$ & $\%$ & $\mathbf{n}$ & $\%$ \\
\hline Católico(a) & 12 & 14,3 & 23 & 27,4 & 35 & 41,7 \\
\hline Evangélico(a) & 26 & 31,0 & 23 & 27,4 & 49 & 58,3 \\
\hline Total & 38 & 45,2 & 46 & 54,8 & 84 & 100,0 \\
\hline
\end{tabular}

Fuente: Cuestionario de factores biosocioculturales y escala de estilo de vida elaborada por: Walker, Sechrist y Pender, modificado por: Diaz, R; Marquéz E; Delgado R. aplicado a las personas adultas mayores del AA.HH. San Juan, febrero 2012. 
Tabla 05

FACTORES SOCIALES Y ESTILO DE VIDA DE LAS PERSONAS ADULTAS MAYORES

\begin{tabular}{|c|c|c|c|c|c|c|}
\hline \multirow{3}{*}{ Estado civil } & \multicolumn{4}{|c|}{ Estilo de vida } & & \\
\hline & \multicolumn{2}{|c|}{ Saludable } & \multicolumn{2}{|c|}{ No saludable } & \multicolumn{2}{|c|}{ Total } \\
\hline & $\mathrm{n}$ & $\%$ & $\mathbf{n}$ & $\%$ & $\mathbf{n}$ & $\%$ \\
\hline Soltero(a) & 3 & 3,6 & 1 & 1,2 & 4 & 4,8 \\
\hline Casado(a) & 8 & 9,5 & 4 & 4,8 & 12 & 14,3 \\
\hline Viudo(a) & 9 & 10,7 & 14 & 16,7 & 23 & 27,4 \\
\hline Unión libre & 16 & 19,0 & 22 & 26,2 & 38 & 45,2 \\
\hline Separado(a) & 2 & 2,4 & 5 & 6,0 & 7 & 8,3 \\
\hline Total & 38 & 45,2 & 46 & 54,8 & 84 & 100,0 \\
\hline
\end{tabular}

\begin{tabular}{|c|c|c|c|c|c|c|}
\hline \multirow{3}{*}{ Ocupación } & \multicolumn{4}{|c|}{ Estilo de vida } & & \\
\hline & \multicolumn{2}{|c|}{ Saludable } & \multicolumn{2}{|c|}{ No saludable } & \multicolumn{2}{|c|}{ Total. } \\
\hline & $\mathrm{n}$ & $\%$ & $\mathbf{n}$ & $\%$ & $\mathbf{n}$ & $\%$ \\
\hline Obrero(a) & 0 & 0,0 & 1 & 1,2 & 1 & 1,2 \\
\hline Ama de casa & 18 & 21,4 & 7 & 8,3 & 25 & 29,8 \\
\hline Agricultor & 8 & 9,5 & 8 & 9,5 & 16 & 19,0 \\
\hline Otros & 12 & 14,3 & 30 & 35,7 & 42 & 50,0 \\
\hline Total & 38 & 45,2 & 46 & 54,8 & 84 & 100,0 \\
\hline
\end{tabular}

Si existe relación significativa.

\begin{tabular}{|c|c|c|c|c|c|c|}
\hline \multirow{3}{*}{$\begin{array}{l}\text { Ingresos } \\
\text { económicos } \\
\text { (soles) }\end{array}$} & \multicolumn{4}{|c|}{ Estilo de vida } & & \\
\hline & \multicolumn{2}{|c|}{ Saludable } & \multicolumn{2}{|c|}{ No saludable } & \multicolumn{2}{|c|}{ Total. } \\
\hline & $\mathrm{n}$ & $\%$ & $\mathbf{n}$ & $\%$ & $\mathbf{n}$ & $\%$ \\
\hline Menos de 100 & 0 & 0,0 & 4 & 4,8 & 4 & 4,8 \\
\hline $100-599$ & 38 & 45,2 & 42 & 50,0 & 80 & 95,2 \\
\hline Total & 38 & 45,2 & 46 & 54,8 & 84 & 100,0 \\
\hline
\end{tabular}

Fuente: Cuestionariode factores biosocioculturales y escala de estilo de vida elaborada por: Walker, Sechrist y Pender, modificado por: Diaz, R; Marquéz E; Delgado R. aplicado a las personas adultas mayores del AA.HH. San Juan, febrero 2012. 


\section{TABLA 04}

Se muestra la relación entre los factores culturales y el estilo de vida. Al relacionar el grado de instrucción con el estilo de vida utilizando el estadístico Chicuadrado de independencia $\left(\chi^{2}=1,384 ; 2 \mathrm{gl}\right.$ y $\left.\mathrm{p}>0,05\right)$, se encuentra que no existe relación significativa entre ambas variables. Así mismo, la relación entre la religión y estilo de vida, haciendo uso del estadístico Chi-cuadrado de independencia $\left(\chi^{2}=2,905 ; 1 \mathrm{gl} \mathrm{y} \mathrm{p}>0,05\right)$, se encontró que no existe relación significativa.

\section{TABLA 05}

$\mathrm{Al}$ relacionar el estado civil con el estilo de vida utilizando el estadístico Chi-cuadrado de independencia $\left(\chi^{2}=4,936 ; 4 \mathrm{gl}\right.$ y $\left.\mathrm{p}>0,05\right)$, se observa que no existe relación significativa entre ambas variables. Así mismo, se muestra la relación entre la ocupación y estilo de vida; haciendo uso del estadístico Chicuadrado de independencia $\left(\chi^{2}=12,909 ; 3 \mathrm{gl} \mathrm{y} \mathrm{p}<0,05\right)$ se encontró que sí existe relación significativa entre las variables. También se muestra la relación entre los ingresos económicos y el estilo de vida, utilizando el estadístico Chicuadrado de independencia $\left(\chi^{2}=3,470 ; 1 \mathrm{gl} \mathrm{y} \mathrm{p}>0,05\right)$, se encontró que no existe relación significativa.

Los resultados muestran que más de la mitad de los adultos mayores del A.H. San Juan tienen estilos de vida no saludables; es decir, sus conductas en relación a la salud no constituye una protección frente a los factores de riesgo. Estos resultados se deben, probablemente, a que los estilos de vida están determinados por factores socioculturales que aprendieron a lo largo de su vida y que permanecen arraigados a pesar del tiempo. Un alto porcentaje proceden de zonas andinas y rurales, donde no se da importancia a las conductas promotoras y protectoras de salud, como la alimentación adecuada, la actividad física, el manejo de estrés, entre otros; por el contrario, sus comportamientos constituyen un riesgo de mortalidad, de morbilidad y de discapacidad funcional en esta etapa de la vida.

Los comportamientos asociados al riesgo o protección de la salud de la persona adulta mayor pueden estar relacionados con el hecho de no haber desarrollado comportamientos preventivos durante su vida; asimismo, debe tenerse en cuenta que los estilos de vida que conducen a problemas de salud no son aspectos que competen únicamente a la responsabilidad y elección del adulto mayor, sino que, en su mayoría, son resultados de los estilos de vida de la sociedad en la que viven.

La OMS ofreció una definición del estilo de vida que lo describía como 
"una forma general de vida basada en la interacción entre las condiciones de vida en un sentido amplio y los patrones individuales de conducta determinados por factores socioculturales y características personales" (WHO, 1986). En el plano ideológico, los estilos de vida se expresan a través de las ideas, valores y creencias que determinan las respuestas o comportamientos a los distintos sucesos de la vida. Desde esta perspectiva integral, los estilos de vida no pueden ser aislados del contexto social, económico, político y cultural, al cual pertenecen y deben ser acordes con los objetivos del proceso de desarrollo que, como fue expuesto, es dignificar la persona humana en la sociedad a través de mejores condiciones de vida y de bienestar. ${ }^{14}$

Según la presente investigación, un poco más de la mitad de los adultos mayores del A.H. San Juan son de sexo masculino. Esto no concuerda con la tendencia de la población a nivel nacional, donde se observa una menor proporción de población masculina que femenina. La mayoría de los adultos mayores tienen grado de instrucción primaria incompleta y completa; esto, debido probablemente a que, por diversos factores, no continuaron con sus estudios para alcanzar un grado más alto. En el contexto social, en el que se desarrollaron, no se pretendía una educación superior; bastaba con la mínima o básica que le permitiera saber leer y escribir, pues su preparación estaba orientada al trabajo. Más de la mitad profesan la religión evangélica; ésto se debe, probablemente, a que actualmente, con la influencia de la globalización y los medios de comunicación, se está dando apertura a nuevos pensamientos e ideologías relacionadas con la religión, las mismas que están ganando adeptos, pues consideran que sus normas, principios y doctrinas son verdaderas y les proporcionan tranquilidad espiritual. ${ }^{15}$

En cuanto a la ocupación, la mitad de los adultos mayores se dedican a otras ocupaciones, considerando entre ellas a ambulantes, comerciantes informales, ligados a la falta de oportunidades laborales y de educación en sus etapas de vidas anteriores; asimismo, la edad en la que se encuentran disminuye aún más sus oportunidades de conseguir un empleo, por lo que están obligados a desempeñar este tipo de trabajos para sentirse útiles a la sociedad y, también, para mantenerse económicamente, pese a que deberían estar descansando, gozando de una jubilación digna, garantizada por el Estado, pero que lamentablemente no alcanza para todos.

Dentro del ingreso económico de los adultos mayores del A.H. San Juan, la mayoría tiene ingreso de 100 a 599 nuevos soles mensuales, ingreso clasificado como económico mínimo para esta población, que no alcanza para cubrir las 
necesidades básicas o para la canasta familiar; esto se debe principalmente al tipo de trabajo que realizan los adultos mayores que no les proporciona mayores ganancias, aumentando así los índices de pobreza a nivel nacional.

Los adultos mayores del A.H. San Juan no presentan una relación significativa entre las variables estilo de vida y el sexo; posiblemente, se deba a que el sexo es la condición o naturaleza orgánica que permite distinguir al varón de la mujer en la especie humana y no determina sus conductas en salud. Hombres y mujeres tienen estilos de vida no saludables, producto de sus propias decisiones, así como por factores socioculturales y económicos. Cabe mencionar que el sexo está determinado por las características genéticas, hormonales, fisiológicas y funcionales, que diferencian biológicamente a los seres humanos. ${ }^{16}$

La mayoría de los adultos mayores tienen grado de instrucción primaria incompleta y presentan estilos de vida no saludables. Los adultos mayores con primaria completa y los analfabetos también presentan estilos de vida no saludables, demostrando que, independientemente del grado de instrucción, los adultos mayores optan por estilos de vida inadecuados debido a que siguen modelos de conducta que aprendieron hace mucho tiempo y que no los perciben como malos o dañinos para su salud.

Existe relación significativa entre el estilo de vida y la ocupación en los adultos mayores del A.H. San Juan. Esto quiere decir, que la ocupación de la población tiene mucho que ver en la adopción de un estilo de vida adecuado o no adecuado, ya que por el tipo de trabajo que desempeñan asumen conductas no saludables, como: no almuerzan a sus horas, o simplemente algo que les pueda saciar el hambre; no realizan actividad física; se encuentran preocupados por la ganancias económicas del día. En ese sentido, la conducta del ser humano es producto de la cultura existente en la sociedad a la que pertenece; por tanto, ésta última determina en gran medida la forma en la que cada persona piensa, cree y

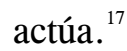

Se observa que no existe relación significativa entre el ingreso económico y el estilo de vida del adulto mayor del AA.HH. San Juan. La mayoría de los adultos mayores tienen un ingreso económico de 100 a 599 nuevos soles y estilos de vida no saludables, un mínimo porcentaje tiene estilos de vida saludables. Estos resultados se deben, probablemente, a que, independientemente de la cantidad de ingresos económicos, los adultos mayores adoptan conductas como respuesta a su interacción con su entorno. Así obtengan mayores ingresos continúan con el mismo patrón de comportamiento en salud, porque esos hábitos lo adquirieron a lo largo de su vida y continúa en el presente. 


\section{CONCLUSIONES}

Existe relación significativa entre el estilo de vida y los factores biosocioculturales.

La mayoría de los adultos mayores presenta un estilo de vida no saludable.

Un poco más de la mitad de los adultos mayores pertenecen al sexo masculino, con un grado de instrucción de primaria incompleta y profesan la religión evangélica. La mayoría tiene estado civil de unión libre, se dedican a otras ocupaciones y tienen un ingreso económico de 100 a 599 nuevos soles.

No existe relación significativa entre el estilo de vida y los factores biosocioculturales: sexo, grado de instrucción, religión, estado civil e ingreso económico.

\section{REFERENCIAS BIBLIOGRÁFICAS}

1 SCIELO - Scientific Electronic Library Online. Concytec - Peru Calle del comercio 197 - San Borja Lima - Perú (Citado el 26 de Noviembre del 2008) Disponible desde el URL: http:www.scielo.org.pe

2 Ministerio DE SALUd "Lineamientos para la dirección general de las personas adultas mayores" 2005 (Citado el 26 de Noviembre del 2008) Disponible desde el URL http:www.upch.edu. pe/vrinve/gerontologia/minsapdf/lineamientos.pdf2

3. NAVARRo, O. Factores de riesgo cardiovascular su repercusión sobre infarto de miocardio y la mortalidad. Instituto de Cardiología del Caribe. Disponible en: http://www.cardiocaribe.com/ newsite/folder/pacientes_factores_riesgo htm.

4 BARDÁLEZ C. La salud en el Perú. [documento en internet]. Consorcio de investigación económica y social. [citado 2010 Ago. 12]. [09 páginas]. Disponible en URL: http://www.bvsde.paho.org/ bvsacd/cd67/saludenelperu.pdf. Descripción de las comunidades que pertenecen a la jurisdicción del Puesto de Salud San Juan. Chimbote 2012 .Oficina de estadística e informática del Puesto de Salud San Juan.

5 MAYA L. Los estilos de vida saludables: componente de la calidad de vida. [Documento en internet]. Colombia; 2001. [Citado 2010 Ago. 12]. [5 páginas]. Disponible en URL: http://www.insp. $\mathrm{mx} /$ Portal/Centros/ciss/promocion_salud/recursos/diplomado/m3/t4/Los_estilos_de_vida_salud ables.pdf

6 ESTEFANERO Y. Estilos de vida de mujeres en la etapa posmenopáusica en el Instituto Nacional Materno Perinatal - [Tesis en internet]. Lima; 2006 [Citado 2010 Ago. 21]. [101 páginas]. Disponible en URL: http://docs.google.com/viewer? $\mathrm{a}=\mathrm{v} \& \mathrm{q}=$ cache: $\mathrm{v}-7 \mathrm{Ka}$ 8Snhq0J:www.cybertesis. edu.pe/sisbib/2007/estefanero_cy/pdf/estefanero_cy.pdf +Estilos + de + vida + de + mujeres + en + la + etapa + posmenop \% C3\% Alusica + en + el + Instituto + Nacional + Materno + Perinatal\&h $\mathrm{l}=\mathrm{es} \& \mathrm{gl}=\mathrm{pe} \& \mathrm{pid}=\mathrm{bl \& srcid}=$ ADGEESjtZgtnwzXxYP9UQxNt9f_sc0f6Epoxhbi7kSxKo0JLV66VZSxJ6zwzTRdPdlPO21-

7 Cid P, MERINo J, StiePOVICH J. Factores biológicos y psicosociales predictores del estilo de vida promotor de salud. [Revista en internet]. Chile; 2006. [Citado 2010 Ago. 13]. [1 pantalla]. Disponible en:http://www.scielo.cl/scielo.php?pid=S0034-98872006001200001\&script $=$ sci_arttext 
8 HERNÁNDEZ R. Metodología de la Investigación. [Articulo en internet]. México: Editorial Mc Graw Hill; 2003. [Citado 2010 Ago. 15]. Disponible en URL: http://www.metabase.net/docs/ unibe/ 03624.html.

9 Wikipedia. Religión. [Artículo en internet]. 2010 [Citado 2010 Set 09]. Disponible en URL: http://es.wikipedia.org/wiki/Religión

10 Definición de Bases de datos on line. Definición de ocupación. [Portada en internet]. 2008. [Citado 2010 Set 09]. Disponible desde el URL: http://definicion.de/ocupacion/

11 Marriner, A y Raile, M. Modelos y Teorías en Enfermería Ed.Mosby; 5 ed. Barcelona.2003.

12 Wikipedia. La enciclopedia libre. Estado civil. [Articulo en internet]. 2010. [Citado 2010 Ago. 28]. [1 pantalla]. Disponible en URL: http://es.wikipedia.org/wiki/Estado_civil

13 Reig F. CABRERo J. Comportamiento y salud en la vejez. Rev, s/r. Geriatric. 1996.

14 Becoña I., VÁsquez, F. \& Oblitas, L. (1999). Promoción de lo Estilos de Vida Saludables: ¿Realidad o Utopía? En: Revista dePsicología de la Pontificia Universidad Católica del Perú. Vol. XVII. 2. 170-228.

15 LOLI J, RonCAL S. Estilo de vida y factores biosocioculturales del la persona adulta mayor del Asentamiento Humano Esperanza Baja. [Trabajo para optar el titulo de licenciatura en enfermería].Chimbote: Universidad los Ángeles de Chimbote; 2009.

16 BOCANEGRA L, INFANTES M. Estilo de vida y factores biosocioculturales del la persona adulta del Asentamiento Humano 10 de Setiembre. [Trabajo para optar el titulo de licenciatura en enfermería]. Chimbote: Universidad los Ángeles de Chimbote; 2009.

17 Mendoza M. Estilo de vida y factores biosocioculturales de la persona adulta del Asentamiento Humano Julio Meléndez. [Trabajo para optar el título de licenciatura en enfermería]. Casma: Universidad Los Ángeles de Chimbote; 2009. 